\title{
THE LAPLACE TRANSFORM OF PAIR DISTRIBUTION FUNCTIONS OF THE MULTICOMPONENT MIXTURE OF DIMERIZING HARD SPHERES
}

\author{
I.A.Protsykevich, M.F.Holovko \\ Institute for Condensed Matter Physics \\ of theUkrainian National Academy of Sciences \\ 1 Svientsitskii St., UA-290011 Lviv-11, Ukraine
}

Received March 5, 1997

\begin{abstract}
The analytical solution of Wertheim's associative Percus-Yevick approximation for the multicomponent mixture of dimerizing hard spheres is analysed. The Laplace transform is obtained for the pair distribution functions.
\end{abstract}

The integral equation theory in multidensity formalism $[1,2,3]$ has been successfully used for the studies of associating fluids [4-11] during the last time. The investigations have, however, focused primarily on the using different numerical iteration schemes. More convenient description of the structure should include the analytical expression on particular for the Laplace transform of pair distribution functions which are also very useful for different applications. In particular, the Laplace transform of the pair distribution functions of pure hard-sphere system was successfully utilized by I.O.Vakarchuk, Yu.K.Rudavskii and co-workers in their theory of amorphous and liquid ferromagnets (see, for example, Ref.[20] and references therein) .

In this paper we have obtain general expressions for the Laplace transform of pair distribution function of the $M$-component system of dimerizing hard spheres. The analytical solution of the associative version of PercusYevick (APY) approximation for this model has been done recently in [5]. For the description of this model it was used the two-density version of the Ornstein-Zernike equation $[1,4,5]$

$$
\left[\tilde{h}_{a b}^{m n}(k)\right]=\left[\tilde{c}_{a b}^{m n}(k)\right]+\left[\tilde{h}_{a b}^{m n}(k)\right] *\left[\sigma_{a b}^{m n}\right] *\left[\tilde{c}_{a b}^{m n}(k)\right],
$$

supplemented by APY closure for the partial pair correlation functions $h_{a b}^{m n}(r)=g_{a b}^{m n}(r)-\delta_{m 0} \delta_{n 0}$ and the partial direct correlation functions $c_{a b}^{m n}(r)$,

$$
\begin{array}{ll}
h_{a b}^{m n}(r)=-\delta_{m 0} \delta_{n 0} & \text { for } r<d_{a b} \\
c_{a b}^{m n}(r)=\delta_{1 m} \delta_{1 n} B_{a b} \delta\left(r-d_{a b}\right) & \text { for } r>d_{a b}
\end{array}
$$

where $d_{a b}=\frac{1}{2}\left(d_{a}+d_{b}\right) ; d_{a}$ is the diameter of particle; $B_{a b}$ are the strength parameters of the stickiness; $\delta(r)$ is the Dirac delta function; $\delta_{m n}$ denotes the Kroneker delta symbol, $g_{a b}^{m n}(r)$ are the partial pair distribution function.

(C) I.A.Protsykevich, M.F.Holovko, 1997

ISSN 0452-9910. Condensed Matter Physics 1997 No 10 (137-142) 137 
The square brackets in (1) represent the matrices, the symbol " $*$ " denotes the convolution and matrix product. The higher indices in the functions $\tilde{h}_{a b}^{m n}(k)$ and $\tilde{c}_{a b}^{m n}(k)$ denote the degree of association of the corresponding particles and can be equal 0 or 1 . The matrix $\left[\sigma_{a b}^{m n}\right]$ is diagonal about the component indices

$$
\sigma_{a b}^{m n}=\sigma_{a}^{m n} \delta_{a b},
$$

where

$$
\sigma_{a}^{00}=\rho_{a}, \quad \sigma_{a}^{01}=\sigma_{a}^{10}=\rho_{a}^{0}, \quad \sigma_{a}^{11}=0 .
$$

The relation between the densities of particles $\rho_{a}$ and monomer densities $\rho_{a}^{0}$ has the following form $[2,5]$

$$
\rho_{a}=\rho_{a}^{0}+4 \pi \rho_{a}^{0} \sum_{b=1}^{M} \rho_{b}^{0}\left(d_{a b}\right)^{2} g_{a b}^{00}\left(d_{a b}^{+}\right) B_{a b} .
$$

The method of solution APY approximation relies on the factorization scheme of Wertheim-Baxter $[12,13]$. As result the solution of equations (1) reduces to the calculation of the corresponding Wertheim-Baxter factor correlation functions which were obtained in [5]. Here we rewrite it in the next compact form

$$
Q_{a b}^{m n}(r)=\left\{\begin{array}{llc}
q_{a b}^{m n}(r), & \text { for } & \lambda_{b a}<r<d_{a b} \\
0 & \text { for } & r<\lambda_{b a} \text { and } r>d_{a b},
\end{array}\right.
$$

where $\lambda_{b a}=\frac{1}{2}\left(d_{b}-d_{a}\right)$,

$$
\begin{aligned}
q_{a b}^{m n}(r) & =q_{a b}^{(0) m n}+\left(r-d_{a b}\right) q_{a b}^{(1) m n}+\frac{1}{2}\left(r-d_{a b}\right)^{2} q_{a b}^{(2) m n} \\
q_{a b}^{(0) m n} & =2 \pi d_{a b} \delta_{1 m} \delta_{1 n} g_{a b}^{00}\left(d_{a b}^{+}\right) B_{a b} \\
q_{a b}^{(1) m n} & =\left(\frac{2 \pi}{\Delta} d_{a b}+\frac{\pi^{2}}{2 \Delta^{2}} \xi_{2} d_{a} d_{b}\right) \delta_{0 m} \delta_{0 n}+\frac{d_{a}}{2} q_{a b}^{(d i m) m n} \\
q_{a b}^{(2) m n} & =\left(\frac{2 \pi}{\Delta}+\frac{\pi^{2}}{\Delta^{2}} \xi_{2} d_{a} d_{b}\right) \delta_{0 m} \delta_{0 n}+q_{a b}^{(d i m) m n}
\end{aligned}
$$

Here

$$
\begin{gathered}
\Delta=1-\frac{\pi}{6} \xi_{3}, \quad \xi_{p}=\sum_{a=1}^{M} \rho_{a}\left(d_{a}\right)^{p}, \\
q_{a b}^{(d i m) m n}=-\frac{4 \pi^{2}}{\Delta} \delta_{0 m} \delta_{1 n} \sum_{c=1}^{M} \rho_{c}^{0} d_{c} d_{c b} g_{c b}^{00}\left(d_{c b}^{+}\right) B_{c b} .
\end{gathered}
$$

The problem of calculating the partial pair distribution functions reduces to the solution of the set of convolution integral equations 


$$
\begin{aligned}
r g_{a b}^{m n}(r) & -\sum_{c=1}^{M} \sum_{l=0}^{1} \sum_{k=0}^{1} \sigma_{c}^{l k} \int_{\lambda_{b a}}^{d_{a b}} \mathrm{~d} t(r-t) g_{a c}^{m l}(|r-t|) Q_{c b}^{k n}(t)= \\
& =\frac{1}{2 \pi}\left(q_{a b}^{(1) m n}+\left(r-d_{a b}\right) q_{a b}^{(2) m n}\right),
\end{aligned}
$$

from which for contact values $g_{a b}^{m n}\left(d_{a b}^{+}\right)$we have

$$
\begin{aligned}
& d_{a b} g_{a b}^{0 n}\left(d_{a b}^{+}\right)=\frac{1}{2 \pi} q_{a b}^{(1) 0 n} \\
& d_{a b} g_{a b}^{1 n}\left(d_{a b}^{+}\right)=\sum_{c=1}^{M} \rho_{c}^{0}\left(\frac{d_{a c}}{\Delta}+\frac{\pi}{4 \Delta^{2}} \xi_{2} d_{a} d_{c}\right) Q_{c b}^{0 n}\left(\lambda_{b c}\right) .
\end{aligned}
$$

By a Laplace transformation, we arrive at the linear algebraic equations

$$
\sum_{c=1}^{M} \sum_{l=0}^{1} \hat{G}_{a c}^{m l}(s)\left(\delta_{c b} \delta_{l n}-\sum_{k=0}^{1} \sigma_{c}^{l k} \hat{Q}_{c b}^{k n}(s)\right)=\frac{\exp \left(-s d_{a b}\right)}{2 \pi s^{2}}\left(q_{a b}^{(2) m n}+s q_{a b}^{(1) m n}\right),
$$

where

$$
\begin{aligned}
\hat{G}_{a b}^{m n}(s) & =\int_{0}^{\infty} \mathrm{d} r r g_{a b}^{m n}(r) \mathrm{e}^{-s r}, \\
\hat{Q}_{a b}^{m n}(s) & =\int_{\lambda_{b a}}^{\infty} \mathrm{d} r Q_{a b}^{m n}(r) \mathrm{e}^{-s r}= \\
& =\exp \left(s \lambda_{a b}\right)\left(\phi_{0}\left(d_{a}\right) q_{a b}^{(0) m n}+\phi_{1}\left(d_{a}\right) q_{a b}^{(1) m n}+\phi_{2}\left(d_{a}\right) q_{a b}^{(2) m n}\right) \\
\phi_{p}\left(d_{a}\right) & =s^{-p-1}\left(\sum_{k=0}^{p} \frac{\left(-s d_{a}\right)^{k}}{k !}-\exp \left(-s d_{a}\right)\right) .
\end{aligned}
$$

Analogically as in $[14,15]$ the solution of eq.(10) reduces in general case to the evaluation of the inverse matrix $W^{-1}$ where the matrix $W$ consists of the element

$$
W_{a b}^{m n}=\delta_{a b} \delta_{m n}-\sum_{k=0}^{1} \sigma_{a}^{m k} \hat{Q}_{a b}^{k n}(s) .
$$

We suppose analogically as in [16], that the coefficient $q_{a b}^{(0) m n}$ can be represented in the form

$$
q_{a b}^{(0) m n}=2 \pi \sigma_{a b} \delta_{1 m} \delta_{1 n} g_{a b}^{00}\left(d_{a b}\right) B_{a b} \equiv\left(w_{a} w_{b}-v_{a} v_{b}\right) \delta_{1 m} \delta_{1 n},
$$


which is the generalization corresponding condition in [17]. Then the matrix $W$ can be written as the Jacobi matrix

$$
W_{a b}^{m n}=\delta_{a b} \delta_{m n}-\hat{a}_{a}^{m} \hat{b}_{b}^{n}-\hat{c}_{a}^{m} \hat{d}_{b}^{n}-\hat{e}_{a}^{m} \hat{f}_{b}^{n}-\hat{z}_{a}^{m} \hat{y}_{b}^{n},
$$

where

$$
\begin{array}{rlrl}
\hat{a}_{a}^{m} & =\frac{\pi}{s \Delta} \sigma_{a}^{m 0}\left(2 \phi_{1}\left(d_{a}\right)+d_{a} \phi_{0}\left(d_{a}\right)\right) ; & \hat{b}_{a}^{m}=\delta_{0 m} ; \\
\hat{c}_{a}^{m} & =\frac{\pi}{2 \Delta} \xi_{2} \hat{a}_{a}^{m}+\frac{\pi}{\Delta} \sigma_{a}^{m 0} \phi_{1}\left(d_{a}\right) ; & \hat{d}_{a}^{m}=d_{a} \delta_{0 m} ; \\
\hat{e}_{a}^{m}=\frac{1}{s} \sigma_{a}^{m 1} w_{a}-P_{w} \hat{a}_{a}^{m} ; & \hat{f}_{a}^{m}=w_{a} \delta_{1 m} ; \\
\hat{z}_{a}^{m}=\frac{1}{s} \sigma_{a}^{m 1} v_{a}-P_{v} \hat{a}_{a}^{m} ; & \hat{y}_{a}^{m}=v_{a} \delta_{1 m} ; \\
P_{w}=\sum_{a=1}^{M} \rho_{a}^{1} d_{a} w_{a} ; & P_{v}=\sum_{a=1}^{M} \rho_{a}^{1} d_{a} v_{a}
\end{array}
$$

After calculation of the inverse matrix $W^{-1}$ we obtain final expression for the Laplace transform of the partial pair distribution functions

$$
\begin{aligned}
& \hat{G}_{a b}^{m n}(s)= \\
& =\frac{\exp \left(-s d_{a b}\right)}{2 \pi s^{2} D_{0}}\left\{( \frac { 2 \pi } { \Delta } \hat { b } _ { a } ^ { m } + \frac { \pi } { \Delta } s \hat { d } _ { a } ^ { m } ) \left((1-\hat{c} \hat{d})\left(\hat{b}_{a}^{n}+E_{1} \hat{h}_{b}^{n}+F_{1} \hat{p}_{b}^{n}\right)+\right.\right. \\
& \left.+(\hat{c} \hat{b})\left(\hat{d}_{b}^{n}+E_{2} \hat{h}_{b}^{n}+F_{2} \hat{p}_{b}^{n}\right)\right)+\left(\frac{\pi^{2}}{\Delta^{2}} \xi_{2} \hat{b}_{a}^{m}+\frac{\pi}{\Delta} s \hat{b}_{a}^{m}+\frac{\pi^{2}}{2 \Delta^{2}} \xi_{2} s \hat{b}_{a}^{m}\right) \times \\
& \left.\quad \times\left((1-\hat{a} \hat{b})\left(\hat{d}_{b}^{n}+E_{2} \hat{h}_{b}^{n}+F_{2} \hat{p}_{b}^{n}\right)+(\hat{a} \hat{d})\left(\hat{b}_{b}^{n}+E_{1} \hat{h}_{b}^{n}+F_{1} \hat{p}_{b}^{n}\right)\right)\right\}+ \\
& +\frac{\exp \left(-s d_{a b}\right)}{2 \pi s^{2} D_{1}}\left\{P_{v}\left(\frac{2 \pi}{\Delta} \hat{b}_{a}^{m}+\frac{\pi}{\Delta} s \hat{d}_{a}^{m}\right)\left((1-\hat{e} \hat{h}) \hat{p}_{b}^{n}+(\hat{e} \hat{p}) \hat{h}_{b}^{n}\right)-\right. \\
& \left.-P_{w}\left(\frac{2 \pi}{\Delta} \hat{b}_{a}^{m}+\frac{\pi}{\Delta} s \hat{d}_{a}^{m}\right)\left((1-\hat{z} \hat{p}) \hat{h}_{b}^{n}+(\hat{z} \hat{h}) \hat{p}_{b}^{n}\right)\right\}
\end{aligned}
$$

where

$$
\begin{aligned}
\hat{h}_{a}^{m} & =\hat{f}_{a}^{m}+\frac{1}{D_{0}}\{(1-\hat{c} \hat{d})(\hat{a} \hat{f})+(\hat{a} \hat{d})(\hat{c} \hat{f})\} \hat{b}_{a}^{m}+ \\
& +\frac{1}{D_{0}}\{(1-\hat{a} \hat{b})(\hat{c} \hat{f})+(\hat{c} \hat{b})(\hat{a} \hat{f})\} \hat{d}_{a}^{m}, \\
\hat{p}_{a}^{m} & =\hat{y}_{a}^{m}+\frac{1}{D_{0}}\{(1-\hat{c} \hat{d})(\hat{a} \hat{y})+(\hat{a} \hat{d})(\hat{c} \hat{y})\} \hat{b}_{a}^{m}+ \\
& +\frac{1}{D_{0}}\{(1-\hat{a} \hat{b})(\hat{c} \hat{y})+(\hat{c} \hat{b})(\hat{a} \hat{y})\} \hat{d}_{a}^{m},
\end{aligned}
$$




$$
\begin{aligned}
& E_{1}= \frac{1}{D_{1}}((1-\hat{z} \hat{p})(\hat{e} \hat{b})+(\hat{e} \hat{p})(\hat{z} \hat{b})), \\
& E_{2}=\frac{1}{D_{1}}((1-\hat{z} \hat{p})(\hat{e} \hat{d})+(\hat{e} \hat{p})(\hat{z} \hat{d})), \\
& F_{1}=\frac{1}{D_{1}}((1-\hat{e} \hat{h})(\hat{z} \hat{b})+(\hat{z} \hat{h})(\hat{e} \hat{b})), \\
& F_{2}=\frac{1}{D_{1}}((1-\hat{e} \hat{h})(\hat{z} \hat{d})+(\hat{z} \hat{h})(\hat{e} \hat{d})), \\
& D_{0}=(1-\hat{a} \hat{b})(1-\hat{c} \hat{d})-(\hat{a} \hat{d})(\hat{c} \hat{b}), \\
& D_{1}=(1-\hat{z} \hat{p})(1-\hat{e} \hat{h})-(\hat{e} \hat{p})(\hat{z} \hat{h}), \\
&(\hat{a} \hat{b})=\sum_{c=1}^{M} \hat{a}_{c} \hat{b}_{c}, \ldots
\end{aligned}
$$

Thus in this letter we have represented the analytical expressions for the Laplace transform of the partial pair distribution functions. From here the expressions for $g_{a b}^{m n}(r)$ can be obtained in explicit form by using zonezone expansions [18] or Laplace transform can be converted to a Fourier transform for which very efficient and very fast programs for calculating the inverse exist. The total pair distribution functions $g_{a b}(r)$ are the sum of four terms

$$
g_{a b}(r)=g_{a b}^{00}+x_{a} g_{a b}^{10}(r)+x_{b} g_{a b}^{01}(r)+x_{a} x_{a} g_{a b}^{11}(r) .
$$

The generality of considered model allows several application of obtained results. Among these we mention the calculation of intercolloidal mean force for which the knowledge of the Laplace transform of the pair distribution functions is very important (see [19] and further citations therein).

The study described in this publication was made in the frames of the Programme for the Fundamental Research State Fund of Ukraine (Code of the grant "associates").

\section{References}

[1] Wertheim M.S. Fluids with highly directional attractive forces. I, II. // J. Stat. Phys., 1984, vol. 35, No 1/2, p. 19-34; 35-47.

[2] Wertheim M.S. Fluids with highly directional attractive forces. III, IV. // J. Stat. Phys., 1986, vol. 42, No 3/4, p. 459-476; 477-492.

[3] Kalyuzhnyi Yu.V., Stell G. On the effects of association in fluids with spherically symmetric interactions. // Mol. Phys., 1993, vol. 78, No 5, p. 1247-1258.

[4] Wertheim M.S. Fluids of dimerizing hard spheres, and fluid mixtures of hard spheres and dispheres. // J. Chem. Phys., 1986, vol. 85, No 5, p. 2929-2936.

[5] Kalyuzhnyi Yu.V., Protsykevich I.A., Holovko M.F. Solution of the associative Percus-Yevick approximation for the n-component mixture of dimerizing hard spheres. // Chem. Phys. Lett., 1993, vol. 215, No 1, p. 1-4.

[6] Wertheim M.S. Percus-Yevick approximation for the Smith-Nezbeda model model of associated fluids. // J. Chem. Phys., 1988, vol. 88, p. 1145-1161. 
[7] Kalyuzhnyi Yu.V., Nezbeda I. Analytical solution of Wertheim's OZ equation for the Smith-Nezbeda model of associated liquids. // Mol. Phys., 1991, vol. 73, p. 703-713.

[8] Holovko M.F., Kalyuzhnyi Yu.V. On the effects of association in the statistical theory of ionic systems. // Mol. Phys., 1991, vol. 73, No 6, p. 1145-1157.

[9] Kalyuzhnyi Yu.V., Holovko M.F., Haymet A.D.J. Integral equation theory for associating liquids; Weakly associated 2-2 electrolytes. // J. Chem. Phys., 1991, vol. 95, No 12, p. 9151-9164.

[10] Protsykevich I.A. General solution of the associative mean spherical approximation for the mixture of dimerizing ions in neutralizing background. // Chem. Phys. Lett., 1996, vol. 252, p. 431-436.

[11] Kalyuzhnyi Yu.V., Vlachy V. Integral equation theory for highly asymmetric electrolyte solutions. // Chem. Phys. Let., 1993, vol. 215, No 5, p. 518-522.

[12] Wertheim M.S. Solution of the Percus-Yevick integral equation. // J. Math. Phys., 1964, vol. 5, No 3, p. 643-654.

[13] Baxter R.J. Ornstein-Zernike relation and Percus-Yevick approximation for fluid mixtures. // J. Chem. Phys., 1970, vol. 52, No 9, p. 4559-4562.

[14] Blum L., Hoye J.S. Mean spherical model for assymetric electrolytes 2. Thermodynamic properties and the pair correlation function. // J. Phys. Chem., 1977, vol. 81, No 13, p. 1311-1316.

[15] Golovko M.F., Protsykevich I.A. Pair correlation functions ffor the assymetric ion-dipole model in the mean spherical approximation. // Chem. Phys. Lett., 1987, vol. 142, No 6, p. 463-468.

[16] Protsykevich I.A. Mean spherical approximation for an arbitrary mixture of ions with surface adhesion in a neutralizing background. // Chem. Phys. Lett., 1995, vol. 232, p. 387-394.

[17] Herrera J.N., Blum L. A solvable model of polydisperse charged particles with sticky interactions. // J. Chem. Phys., 1991, vol. 94, p. 6190-6195.

[18] Smith W.R., Henderson D.J. Analytical representation of the Percus-Yevick hard sphere radial distribution function. // Mol. Phys., 1970, vol. 19, No 3, p. 411-416.

[19] Holovko M.F., Trokhymchuk A.D., Protsykevich I.A., Henderson D.J. The Laplace transform of the MSA pair distribution functions between macroions in a ion-dipole fluid. // J. Stat. Phys., 1993, vol. 72, No 5/6, p. 1391-1399.

[20] Vakarchuk I.O., Rudavsky Yu.K., Ponedilok J.V. About the problem of magnetizm in topological non-odering system. // In Problem of modern statystical physics. Ed. by N.N.Bogolubov, Kiev, Naukova dumka, 1985, p. 63-72.

\title{
ЛАПЛАС-ОБРАЗ ПАРНИХ ФУНКЦІЙ РОЗПОДІЛУ БАГАТОКОМПОНЕНТНОЇ СУМІШІ ТВЕРДИХ СФЕР, ЩО ДИМЕРИЗУЮТЬСЯ
}

\author{
І.А.Процикевич, М.Ф.Головко
}

\begin{abstract}
У роботі представлено вираз для лаплас-образів парних функцій розподілу багатокомпонентної суміші димеризуючих твердих сфер. Розрахунки зроблені на основі отриманого нами раніше аналітичного розв'язку інтегральних рівнянь типу Орнштейна-Церніке (теорія асоціативних рідин Вертхайма) в наближенні Перкуса-Йевіка.
\end{abstract}

